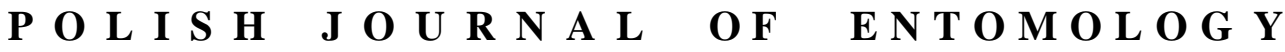

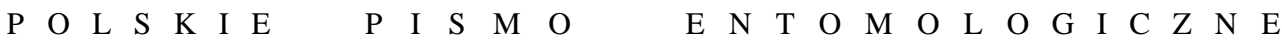

VOL. 84: $383-420$

Lublin

30 December 2015

DOI: $10.1515 /$ pjen-2015-0033

\section{An annotated checklist of the Aphids (Hemiptera: Aphidomorpha) of Poland}

\section{WACŁAW WOJCIECHOWSKI, ŁUKASZ DEPA, MARIUSZ KANTURSKI, PIOTR WEGIEREK, KARINA WIECZOREK}
Department of Zoology, Faculty of Biology and Environmental Protection, University of Silesia, Bankowa 9, 40-007 Katowice, Poland,

e-mails: waclaw.wojciechowski@us.edu.pl, lukasz.depa@us.edu.pl,mkanturski@us.edu.pl, piotr.wegierek@us.edu.pl, karina.wieczorek@us.edu.pl

\begin{abstract}
The paper presents a comprehensive compilation of 764 taxa (species and subspecies), distributed over 167 genera, belonging to 17 subfamilies, three families and three superfamilies of Aphidomorpha recorded to date from Poland. The systematic positions of 19 taxa have been revised in accordance with recent changes in nomenclature. The presence in the Polish aphidofauna of Drepanosiphum oregonensis and Coloradoa huculaki, previously included without any distribution data in checklists of Polish aphids, has been confirmed. One species Sitobion (Sitobion) alopecuri is recognized as being new to Poland. At least 44 species (6\% of local fauna of Aphidomorpha) are alien to Poland; among them 11 species collected from plants imported or cultivated in indoor conditions are listed.
\end{abstract}

KEY WORDS: aphids, new species, systematic list, taxonomy.

\section{INTRODUCTION}

Following the publication of the Catalogue of Polish Fauna - Aphidoidea by SzELEGIEWICZ (1968), three checklists of Polish species of aphids (Hemiptera: Aphidomorpha) by SZELEGIEWICZ (1978a), NAST et al. (1990) and WEGIEREK \& WOJCIECHOWSKI (2004) were issued. Recently, a Distributional Checklist of the Aphids of Poland by OsIADACZ \& HAŁAJ (2009) was published, supplemented by a Systematic Review of the Aphids of Poland with a Host Plant Index (OSIADACZ \& HAŁAJ 2010) and an update of the latter work (OSIADACZ \& HAŁAJ 2012). 
As there were still errors and omissions in those earlier papers, a new and comprehensive compilation of the Polish Aphidomorpha in the form of checklist was deemed necessary in the light of recent systematic and nomenclatural revisions.

In the present paper, updated information is provided on the systematic positions and nomenclatural changes of 19 taxa. Based on available specimens and taxonomic analysis, one species (Sitobion (Sitobion) alopecuri (TAKAHASHI, 1921)) is accepted as being new to Poland. The presence in the Polish aphidofauna of two species Drepanosiphum oregonensis GRANOVSKY, 1939 and Coloradoa huculaki SZELEGIEWICZ, 1981, previously included without any distribution data in the systematic reviews of Polish aphids by SzELEGIEWICZ (1978a), NAST et al. (1990), WEGIEREK \& WOJCIECHOWSKI (2004) and OSIADACZ \& HAŁAJ $(2009,2010)$, has now been confirmed. However, nine taxa listed in the above-mentioned papers - Eriosoma grossulariae (SCHÜLE, 1887); Anoecia (Anoecia) major BÖRNER, 1950; Aphis frangulae testacea K.H. THOMAS, 1968; Cryptomyzus galeopsidis dickeri HILLE RIS LAMBERS, 1953; C. galeopsidis galeopsidis (KALTENBACH, 1843); Macrosiphoniella (Asterobium) linariae (KOCH, 1855); M. (Macrosiphoniella) kaufmanni BÖRNER, 1940; Ovatomyzus boraginacearum EASTOP, 1952; Sitobion (Sitobion) paludum MÜLLER, 1982 still require such confirmation. These taxa are included in the present work but are not numbered.

As a consequence of the increasing globalisation of trade, there is a strong potential for the introduction of alien species into the native fauna. Therefore, Adelgidae (five species), Phylloxeridae (one species) and Aphididae (38 species) alien to Poland are indicated with an exclamation mark in the checklist. Polyphagous species of aphids, colonising plants cultivated in glasshouses, can occasionally infest plants in natural habitats. Therefore, species collected from plants imported or cultivated indoors have been added and indicated in the checklist (11 species).

Consequently, the list includes 764 taxa (species and subspecies) belonging to 167 genera representing 17 subfamilies, 3 families and 3 superfamilies of Aphidomorpha. Adelgidae is represented by 13 species, Phylloxeridae by 6 species and Aphididae by 745 taxa of specific or subspecific rank: Eriosomatinae 47, Hormaphidinae 3, Anoeciinae 6, Thelaxinae 5, Mindarinae 2, Drepanosiphinae 4, Phyllaphidinae 1, Calaphidinae 42, Saltusaphidinae 21, Chaitophorinae 38, Aphidinae 524 (Aphidini 166, Macrosiphini 358), Lachninae 51.

\section{MATERIAL AND METHODS}

The names and classification follow NIETO NAFRía \& FAVRET (2011), with the exception of the taxonomic position of all the former Pterocommatinae, which have been 
placed in the tribe Macrosiphini. The positions of questionable taxa were verified according to García Prieto et al. (2004), Nieto Nafría et al. (2009), Blackman (2010), BLACKMAN \& EASTOP $(2011,2015)$. To extend the register of alien species present in Poland (WIECZOREK 2011), we used the list of Alien European Aphididae compiled by COEUR D'ACIER et al. (2010). Adelgidae and Phylloxeridae alien to Poland have been listed according to OSIADACZ \& HALAJ (2011) and SOIKA \& LABANOWSKI (2014); they are indicated with an exclamation mark in the checklist.

Abbreviations: UŚ - Department of Zoology, University of Silesia, Katowice, Poland; the examined slides are deposited in this institution; 1-764 - the numbers in the list refer to taxa of specific and subspecific rank; ! - species alien to Poland; * - species new to Poland;

** - species recorded from imported plants or cultivated in indoor conditions; ${ }^{1-21}-$ the numbers in the list refer to taxonomic comments.

The names of particular subgenera and taxa of lower rank within genera have been arranged in alphabetical order.

THE APHIDS (HEMIPTERA: APHIDOMORPHA) OF POLAND: A CHECKLIST

Order: HEMIPTERA LINNAEUS, 1758

Suborder: STERNORRHYNCHA AMYOT \& SERVILLE, 1843

Infraorder: APHIDOMORPHA BEKKER-MIGDISOVA \& AIZENBERG, 1991

Superfamily: ADELGOIDEA ANNAND, 1928

Family: ADELGIDAE ANNAND, 1928

Subfamily: ADELGINAE ANNAND, 1928

Genus: Adelges VALLOT, 1836

Subgenus: Adelges VALLOT, 1836

Species: laricis VALLOT, 1836 tardus (DREYFUS, 1888)

Subgenus: Aphrastasia BÖRNER, 1909

Species: pectinatae (CHOLODKOVSKY, 1888)!

Sugenus: Dreyfusia BÖRNER, 1908

Species: nordmannianae (ECKSTEIN, 1890)! piceae (RATZEBURG, 1844)

Subgenus: Gilletteella BÖRNER, 1930

Species: cooleyi (GILLETTE, 1907)!

Subgenus: Sacchiphantes CURTIS, 1844

Species: abietis (LINNAEUS, 1758)

viridis (RATZEBURG, 1843) 
Subfamily: PINEINAE NÜSSLIN, 1909

Genus: Pineus SHIMER, 1869

9

10

11

12

13

14

15

16

17

18

19

Species: cembrae (CHOLODKOVSKY, 1888) orientalis (DREYFUS, 1889)! pineoides (CHOLODKOVSKY, 1903) pini (RATZEBURG, 1844) strobi (HARTIG, 1837) !

Superfamily: PHYLLOXEROIDEA HERRICH-SCHAEFFER, 1854

Family: PHYLLOXERIDAE HERRICH-SCHAEFFER, 1854

Subfamily: PHYLLOXERINAE HERRICH-SCHAEFFER, 1854

Tribe: Acanthochermesini BÖRNER, 1913

Genus: Acanthochermes KOLLAR, 1848

Species: quercus KOLlaR, 1848

Tribe: Phylloxerini HERRICH-SCHAEFFER, 1854

Genus: Daktulosphaira SHIMER, $1866^{1}$ Species: vitifoliae (FITCH, 1851) !

Genus: Phylloxera BOYER DE FONSCOLOMBE, 1834 Species: coccinea (VON HEYDEN, 1837) glabra (VON HEYDEN, 1837)

Subfamily: PHYLLOXERININAE BÖRNER, 1908

Genus Phylloxerina BÖRNER, 1908

Species: capreae BÖRNER, 1942 salicis (LICHTENSTEIN, 1884)

Superfamily: APHIDOIDEA LATREILLE, 1802

Family: APHIDIDAE LATREILLE, 1802

Subfamily: ERIOSOMATINAE KIRKALDY, 1905

Tribe: Eriosomatini KIRKALDY, 1905

Genus: Colopha MONELL, 1877

Species: compressa (KoCH, 1856)

Genus: Eriosoma LEACH, 1818

Species: anncharlotteae DANIELSSON, 1979 grossulariae (SCHÜLE, 1887) lanigerum (HAUSMANN, 1802) !

lanuginosum (HARTIG, 1839)

patchiae (BÖRNER \& BLUNCK, 1916)

ulmi ulmi (LINNAEUS, 1758) 
Genus: Kaltenbachiella SCHOUTEDEN, 1906 Species: pallida (HALIDAY, 1838)

Genus: Tetraneura HARTIG, 1841

Subgenus: Tetraneura HARTIG, 1841

Species: ulmi (LINNAEUS, 1758) africana VAN DER GOOT, 1912

Subgenus: Tetraneurella HILLE RIS LAMBERS, 1970 Species: nigriabdominalis (SASAKI, 1899)**

Tribe: Fordini ACLOQUE, 1897

Genus: Aploneura PASSERINI, 1863

Species: lentisci (PASSERINI, 1856)

Genus: Baizongia RONDANI, 1848

Species: pistaciae (LINNAEUS, 1767)

Genus: Forda VON HEYDEN, 1837

Species: formicaria VON HEYDEN, 1837

marginata $\mathrm{KOCH}, 1857$

pawlowae MORDVILKO, 1901

Genus: Geoica HART, 1894

Species: setulosa (PASSERINI, 1860) utricularia (PASSERINI, 1856)

Genus: Paracletus vON HEYDEN, 1837

Species: bykovi bykovi (MORDVILKO, 1921) cimiciformis cimiciformis VON HEYDEN, 1837

Genus: Smynthurodes WESTWOOD, 1849

Species: betae WESTWOOD, 1849

Tribe: Pemphigini HERRICH-SCHAEFFER, 1854

Genus: Gootiella TULLGREN, 1925

Species: alba SHAPOSHNIKOV, 1952 tremulae TULLGREN, 1925

Genus: Mimeuria BÖRNER, 1952

Species: ulmiphila (DEL GUERCIO, 1917)

Genus: Mordwilkoja DEL GUERCIO, 1909

Species: vagabunda (WALSH, 1863)

Genus: Pachypappa KoCH, 1856

Species: marsupialis marsupialis KоCH, 1856

populi (LINNAEUS, 1758)

tremulae (LINNAEUS, 1761)

vesicalis $\mathrm{KOCH}, 1856$ 
Genus: Pemphigus HARTIG, 1839

Subgenus: Pemphigus HARTIG, 1839

Species: betae DOANE, 1900

borealis TULLGREN, 1909

bursarius (LINNAEUS, 1758)

fuscicornis (KOCH, 1857)

immunis BUCKTON, 1896

phenax BÖRNER \& BLUNCK, 1916

populi COURCHET, 1879

populinigrae (SCHRANK, 1801)

protospirae LICHTENSTEIN, 1885

spyrothecae PASSERINI, 1856

Genus: Prociphilus KOCH, 1857

Subgenus: Prociphilus KoCH, 1857

Species: bumeliae (SCHRANK, 1801)

fraxini (FABRICIUS, 1777)

Subgenus: Stagona KoCH, 1857

Species: pini (BURMEISTER, 1835)

xylostei xylostei (DE GEER, 1773)

Genus: Thecabius KoCH, 1857

Subgenus: Parathecabius BÖRNER, 1950

Species: lysimachiae BÖRNER, 1916

Subgenus: Thecabius KoCH, 1857

Species: affinis (KALTENBACH, 1843)

Subfamily: HORMAPHIDINAE MORDVILKO, 1908

Tribe: Cerataphidini BAKER, 1920

Genus: Cerataphis LICHTENSTEIN, 1882

Species: lataniae (BOISDUVAL, 1867)**!

Tribe: Hormaphidini MORDVILKO, 1908

Genus: Hamamelistes SHIMER, 1867 
Genus: Hormaphis OSTEN-SACKEN, 1861

69 Species: betulae (MORDVILKO, 1901)

Subfamily: PHLOEOMYZINAE MORDVILKO, 1934

Genus: Phloeomyzus HORVÁTH, 1896

Subfamily: ANOECIINAE TULLGREN, 1909

Genus: Anoecia KoCH, 1857

Subgenus: Anoecia KoCH, 1857

71

72

73

74

75

76

77

78

79

80

81

82

83

84

85

86
Species: corni (FABRICIUS, 1775)

furcata (THEOBALD, 1915)

major BÖRNER, 1950

stipae MAMONTOVA, 1968

vagans (KOCH, 1856)

zirnitsi MORDVILKO, 1931

Subgenus: Paranoecia ZWÖLFER, 1957

Species: pskovica MORDVILKO, 1921

Subfamily: THELAXINAE BAKER, 1920

Genus: Glyphina КоCH, 1856

Species: betulae (LINNAEUS, 1758)

jacutensis MORDVILKO, 1931

pseudoschrankiana BLACKMAN, 1989

Genus: Thelaxes Westwood, 1840

Species: dryophila (SCHRANK, 1801) suberi (DEL GUERCIO, 1911)

Subfamily: MINDARINAE TULLGREN, 1909

Genus: Mindarus KoCH, 1857

Species: abietinus $\mathrm{KoCH}, 1857$

obliquus (CHOLODKOVSKY, 1896)

Subfamily: DREPANOSIPHINAE HERRICH-SCHAEFFER, 1857

Genus: Drepanosiphum KOCH, 1855

Species: acerinum (WALKER, 1848)

aceris $\mathrm{KOCH}, 1855$

oregonensis GRANOVSKY, $1939^{2}$ 
Subfamily: PHYLLAPHIDINAE HERRICH-SCHAEFFER, 1857

Genus: Phyllaphis KOCH, 1856

Subfamily: CALAPHIDINAE OESTLUND, 1919

Tribe: Calaphidini OESTLUND, 1919

Genus: Betulaphis GLENDENNINg, 1926 quadrituberculata (KALTENBACH, 1843)

Genus: Boernerina BRAMSTEDT, 1940

Species: depressa BRAMSTEDT, 1940

Genus: Calaphis WALSH, (1862) 1863

Species: betulicola (KALTENBACH, 1843) flava MORDVILKO, 1928

Genus: Callipterinella VAN DER GOOT, 1913

Species: calliptera (HARTIG, 1841) tuberculata (VON HEYDEN, 1837)

Genus: Clethrobius MORDVILKO, 1928

Species: comes (WALKER, 1848)

Genus: Euceraphis WALKER, 1870

Species: betulae (KoCH, 1855) punctipennis (ZETTERSTEDT, 1828)

Genus: Monaphis WALKER, 1870

Genus: Symydobius MORDVILKO, 1894

Subgenus: Symydobius MORDVILKO, 1894

Species: oblongus (VON HEYDEN, 1837)

Tribe: Panaphidini Oestlund, 1923

Genus: Appendiseta RICHARDS, 1965

Genus: Chromaphis WALKER, 1870

Genus: Ctenocallis KLODNITSKY, 1924 
Genus: Myzocallis PASSERINI, 1860

Subgenus: Agrioaphis WALKER, 1870

105

Species: castanicola BAKER, 1917

106

myricae (KALTENBACH, 1843)

107

Subgenus: Lineomyzocallis RICHARDS, 1965

Species: walshii (MONELL in RILEY \& MONELL, 1879) !

108

Subgenus: Myzocallis PASSERINI, 1860

Species: boerneri STROYAN, 1957

109

carpini (KOCH, 1855)

110

coryli (GOEZE, 1778)

Genus: Panaphis KIRKALDY, 1904

111

Species: juglandis (GOEZE, 1778) !

Genus: Pterocallis PASSERINI, 1860

Subgenus: Pterocallis PASSERINI, 1860

112

Species: albidus BÖRNER, 1940

alni (DE GEER, 1773)

maculata (VON HEYDEN, 1837)

Genus: Therioaphis WALKER, 1870

Subgenus: Pterocallidium BÖRNER, 1949

Species: luteola (BÖRNER, 1949)

subalba BÖRNER, 1949

trifolii (MONELL, 1882)

117

Subspecies: trifolii (MONELL, 1882)

ventromaculata F. P. MÜLLER, 1968

Subgenus: Rhizoberlesia DEL GUERCIO, 1919

Species: brachytricha HILLE RIS LAMBERS \& VAN DEN

BosCH, 1964

120

riehmi (BÖRNER, 1949)

Subgenus: Therioaphis WALKER, 1870

121

Species: ononidis ononidis (KALTENBACH, 1846)

122

tenera tenera (AIZENBERG, 1956)

Genus: Tinocallis MATSUMURA, 1919

Subgenus: Eotinocallis QUEDNAU, 2003

Species: platani (KALTENBACH, 1843)

Subgenus: Sappocallis MATSAMURA, 1919 
Genus: Tuberculatus MORDVILKO, 1894

Subgenus: Tuberculatus MORDVILKO, 1894

126

127

128

129

130
Species: querceus (KALTENBACH, 1843)

Subgenus: Tuberculoides VAN DER GOOT, 1915

Species: annulatus (HARTIG, 1841)

borealis (KRZYWIEC, 1971)

eggleri BÖRNER, 1950

neglectus (KRZYWIEC, 1966)

Subfamily: SALTUSAPHIDINAE BAKER, 1920

Tribe: Saltusaphidini BAKER, 1920

Genus: Iziphya NEVSKY, 1929

Species: bufo (WALKER, 1848) ingegardae HILLE RIS LAMBERS, 1952 memorialis BÖRNER, 1950

Genus: Juncobia QUEDNAU, 1954 Species: leegei (BÖRNER, 1940) Genus: Nevskya OssIANNILSSON, 1953 Species: fungifera OSSIANNILSSON, 1953

Genus: Saltusaphis THEOBALD, 1915

Species: scirpus THEOBALD, 1915

Genus: Sminthuraphis QUEDNAU, 1953

Species: ulrichi QUEDNAU, 1953

Tribe: Thripsaphidini BÖRNER, 1949

Genus: Allaphis MORDVILKO, 1921

Species: cyperi (WALKER, 1848) ossiannilssoni (HILLE RIS LAMBERS, 1952) producta (GILLETTE, 1917) verrucosa verrucosa (GILLETTE, 1917)

Genus: Subsaltusaphis QUEDNAU, 1953

Subgenus: Subsaltusaphis QUEDNAU, 1953

Species: flava (HILLE RIS LAMBERS, 1939) intermedia (HILLE RIS LAMBERS, 1939) lambersi lambersi (QUEDNAU, 1954) ornata (THEOBALD, 1927) pallida (HILLE RIS LAMBERS, 1939) paniceae (QUEDNAU, 1954) picta (HILLE RIS LAMBERS, 1939) 
rossneri (BÖRNER, 1940)

Genus: Thripsaphis GILLETTE, 1917

Subgenus: Neobacillaphis HUCULAK, 1968

Species: szelegiewiczi szelegiewiczi (HUCULAK, 1968)

Subfamily: CHAITOPHORINAE MORDVILKO, 1908

Tribe: Chaitophorini MORDVILKO, 1908

Genus: Chaitophorus KoCH, 1854

Species: capreae (MosLEY, 1841) diversisetosus diversisetosus SZELEGIEWICZ \& CZYLOK, 1981 horii (TAKAHASHI, 1939)

Subspecies: horii beuthani (BÖRNER, 1950) hypogaeus HILLE RIS LAMBERS, 1947 leucomelas KOCH, 1854 longisetosus SZELEGIEWICZ, 1959 mordvilkoi MAMONTOVA, 1961 nassonowi MORDVILKO, 1894 parvus HILLE RIS LAMBERS, 1935 pentandrinus OSSIANNILSSON, 1959 populeti populeti (PANZER, 1804) populialbae populialbae (BOYER DE FONSCOLOMBE, 1841) ramicola (BÖRNER, 1949) salicti (SCHRANK, 1801) salijaponicus ESSING \& KUWANA, 1918

Subspecies: salijaponicus niger MORDVILKO, 1929 tremulae tremulae $\mathrm{KoCH}, 1854$ truncatus (HAUSMANN, 1802) vitellinae vitellinae (SCHRANK, 1801)

Genus: Periphyllus VAN DER HOEVEN, 1863

Species: acericola (WALKER, 1848) aceris (LINNAEUS, 1761) californiensis californiensis (SHINJI, 1917) ! coracinus (KOCH, 1854) hirticornis (WALKER, 1848) 
175

176

177

178

179

180

181

182

183

184

185

186

187

188

189

190

191

192

193

194

195

196

197

198 lyropictus (KESSLER, 1886)

obscurus MAMONTOVA, 1955

testudinaceus (FERNIE, 1852)

Tribe: Siphini MORDVILKO, 1928

Genus: Atheroides HALIDAY, 1839

Species: doncasteri OSSIANNILSSON, 1955

hirtellus HALIDAY, 1839

serrulatus HALIDAY, 1839

Genus: Caricosipha BÖRNER, 1939

Species: paniculatae BÖRNER, 1939

Genus: Chaetosiphella HILLE RIS LAMBERS, 1939

Species: berlesei (DEL GUERCIO, 1905)

stipae stipae HILLE RIS LAMBERS, 1947

tshernavini (MORDVILKO, 1921)

Genus: Laingia THEOBALD, 1922

Species: psammae THEOBALD, 1922

Genus: Sipha PASSERINI, 1860

Subgenus: Sipha PASSERINI, 1860

Species: glyceriae (KALTENBACH, 1843)

Subgenus: Rungsia MIMEUR, 1933

Species: arenarii MORDVILKO, 1921

elegans Del Guercio, 1905

maydis PASSERINI, 1860

Subfamily: APHIDINAE LATREILLE, 1802

Tribe: Aphidini LATREILLE, 1802

Genus: Anthemidaphis TASHEV, 1967

Species: oligommata (TASHEV, 1967)

Genus: Aphis LinNaEus, 1758

Subgenus: Aphis LINNAEUS, 1758

Species: acanthoidis (BÖRNER, 1940)

acetosae LINNAEUS, 1761

affinis DEL GUERCIO, 1911

althaeae althaeae (NEVSKY, 1929) ${ }^{3}$

antherici HOLMAN, 1966

austriaca HILLE RIS LAMBERS, 1950

ballotae (PASSERINI, 1860) ${ }^{4}$

berlinskii HUCULAK, 1968 
199

200

201

202

203

204

205

206

207

208

209

210

211

212

213

214

215

216

217

218

219

220

221

222

223

224

225

226

227

228

229

230

231

232 berteroae SZELEGIEWICZ, 1966

breviseta HOLMAN, 1966

brohmeri BÖRNER, 1952

brunellae SCHOUTEDEN, 1903

bupleuri (BÖRNER, 1932)

cacaliasteris HILLE RIS LAMBERS, 1947

calaminthae (BÖRNER, 1952)

callunae THEOBALD, 1915

cerastii (BÖRNER, 1950)

chloris $\mathrm{KOCH}, 1854$

cirsiioleracei (BÖRNER, 1932)

clematidis clematidis $\mathrm{KOCH}, 1854$

clinopodii PASSERINI, 1861

comari PRIOR \& STROYAN, 1977

commensalis STROYAN, 1952

comosa (BÖRNER, 1950)

confusa WALKER, 1849

coronillae FERRARI, 1872

Subspecies: arenaria HOFFMANN, 1968 coronillae FERRARI, 1872

craccae LINNAEUS, 1758

craccivora craccivora $\mathrm{KoCH}, 1854$

crepidis (BÖRNER, 1940)

cytisorum HARTIG, 1841

Subspecies: cytisorum HARTIG, 1841 sarothamni FRANSSEN, 1928

eryngiiglomerata BOZHKO, 1963

etiolata STROYAN, 1952

euphorbiae KALTENBACH, 1843

fabae SCOPOLI, 1763

Subspecies: cirsiiacanthoidis SCOPOLI, 1763

evonymi FABRICIUS, 1775

fabae SCOPOLI, 1763

solanella THEOBALD, 1914

farinosa farinosa GMELIN, 1790

forbesi WEED, 1889 !

frangulae KALTENBACH, 1845

Subspecies: frangulae KALTENBACH, 1845 
beccabungae $\mathrm{KoCH}, 1855$

testacea K. H. THOMAS, 1968

funitecta (BÖRNER, 1950)

galiiscabri SCHRANK, 1801

genistae SCOPOLI, 1763

gentianae (BÖRNER, 1940)

gossypii GLOVER, 1877

Subspecies: capsellae KALTENBACH, 1843 gossypii GLOVER, 1877** !

hederae KALTENBACH, 1843

helianthemi helianthemi FERRARI, 1872

hieracii SCHRANK, 1801

hypochoeridis (BÖRNER, 1940)

idaei VAN DER GOOT, 1912

ilicis KALTENBACH, 1843

intybi KOCH, 1855

jacobaeae SCHRANK, 1801

janischi (BÖRNER, 1940)

klimeschi (BÖRNER, 1950)

korshunovi IVANOVSKAJA, 1971

lambersi (BÖRNER, 1940)

lamiorum (BÖRNER, 1950)

leontodontis (BÖRNER, 1950)

longini HUCULAK, 1968

loti KALTENBACH, 1862

lotiradicis STROYAN, 1972

mammulata GHIMINGHAM \& HILLE RIS LAMBERS, 1949

mamonthovae DAVLETSHINA,1964

medicaginis $\mathrm{KoCH}, 1854$

mirifica (BÖRNER, 1950)

molluginis (BÖRNER, 1950)

montanicola HILLE RIS LAMBERS, 1950

nasturtii KALTENBACH, 1843

nerii BOYER DE FONSCOLOMBE, 1841**

newtoni THEOBALD, 1927

origani PASSERINI, 1860

pilosellae (BÖRNER, 1952) 
268

269

270

271

272

273

274

275

276

277

278

279

280

281

282

283

284

285

286

287

288

289

290

291

292

293

294

295

296

297

298

299

300

301

302

303

304 picridis (BÖRNER, 1950) ${ }^{5}$

plantaginis GOEZE, 1778

podagrariae SCHRANK, 1801

pollinaria (BÖRNER, 1952)

polygonata (NEVSKY, 1929)

pomi DE GEER, 1773

praeterita WALKER, 1849

proffti (BÖRNER, 1942)

psammophila SZELEGIEWICZ, 1967

pseudocomosa STROYAN, 1972

pulsatillicola HOLMAN, 1966

roepkei (HILLE RIS LAMBERS, 1931)

ruborum (BÖRNER \& SCHILDER, 1931)

rumicis LINNAEUS, 1758

salicariae $\mathrm{KOCH}, 1855$

salviae WALKER, 1852

sambuci LINNAEUS, 1758

sanguisorbae SCHRANK, 1801

Subspecies: poterii (BÖRNER, 1940)

sanguisorbae SCHRANK, 1801

schilderi (BÖRNER, 1940)

sedi KALTENBACH, 1843

selini (BÖRNER, 1940)

serpylli $\mathrm{KOCH}, 1854$

seselii (BOZHKO, 1959)

spiraecola $\mathrm{PATCH}, 1914 * *$ !

spiraephaga MÜLLER (F.P.), 1961 !

stachydis MORDVILKO, 1929

subnitida (BÖRNER, 1940)

subviridis (BÖRNER, 1940)

symphyti SCHRANK, 1801

tacita HUCULAK, 1968

taraxacicola (BÖRNER, 1940)

teucrii (BÖRNER, 1942)

thalictri thalictri $\mathrm{KOCH}, 1854$

thesii HOLMAN, 1966

thomasi (BÖRNER, 1950)

tormentillae PASSERINI, 1879 
305

306

307

308

309

310

311

312

313

314

315

316

317

318

319

320

321

322 triglochinis THEOBALD, 1926

ulmariae SCHRANK, 1801

umbrella (BÖRNER, 1950)

urticata GMELIN, 1790

uvaeursi OSSIANNILSSON, 1959

vaccinii (BÖRNER, 1940)

veratri WALKER, 1852

verbasci SCHRANK, 1801

verticillatae (BÖRNER, 1940)

viburni SCOPOLI, 1763

vineti HOFFMANN, 1972

violae SCHOUTEDEN, 1900

vitalbae FERRARI, 1872

Subgenus: Bursaphis MAC VICAR BAKER, 1934

Species: epilobii KALTENBACH, 1843

grossulariae KALTENBACH, 1843

holoenotherae RAKAUSKAS, 2007

oenotherae oenotherae OESTLUND, 1887 !

schneideri (BÖRNER, 1940)

Subgenus: Toxoptera $\mathrm{KOCH}, 1856$

Species: aurantii aurantii BOYER DE FONSCOLOMBE, $1841 * *$ !

Genus: Hyalopterus KOCH, 1854

Species: amygdali (E. BLANCHARD, 1840) pruni (GEOFFROY, 1762)

Genus: Melanaphis VAN DER GOOT, 1917

Species: luzulella (HILLE RIS LAMBERS, 1947) pyraria (PASSERINI, 1861)

Genus: Protaphis BÖRNER, 1952

Species: anthemidis (BÖRNER, 1940) carlinae (BÖRNER, 1940) elongata (NEVSKY, 1928) erigerontis HOLMAN, 1966 hartigi (HILLE RIS LAMBERS, 1931) picridicola HOLMAN, 1966

Genus: Rhopalosiphum KoCH, 1854

Species: maidis (FITCH, 1856) ! nymphaeae (LINNAEUS, 1761) 
336

337

338

339

340

341

342

344

345

346

347

348

349

350

351

352

353

354

355

356

357

358

359

360

\author{
oxyacanthae (SCHRANK, 1801) ! \\ padi (LINNAEUS, 1758) \\ rufiabdominale (SASAKI, 1899) ${ }^{6} * *$ ! \\ rufulum RICHARDS, 1960
}

Genus: Schizaphis BÖRNER, 1931

Subgenus: Euschizaphis HILLE RIS LAMBERS, 1947

Species: palustris (THEOBALD, 1929)

Subgenus: Paraschizaphis HILLE RIS LAMBERS, 1947

Species: caricis (SCHOUTEDEN, 1906) scirpi scirpi (PASSERINI, 1874)

Subgenus: Schizaphis BÖRNER, 1931

Species: agrostis HILLE RIS LAMBERS, 1947

dubia HUCULAK, 1968

graminum (RONDANI, 1852)

holci HILLE RIS LAMBERS, 1947

jaroslavi (MORDVILKO, 1921)

longicaudata HILLE RIS LAMBERS, 1939

nigerrima (HILLE RIS LAMBERS, 1931)

rotundriventris (SIGNORET, 1860) **

rufula (WALKER, 1849)

wahlgreni (OSSINNILSSON, 1959)

weingaertneriae HILLE RIS LAMBERS, 1947

Genus: Toxopterina BÖRNER, 1940

Species: vandergooti (BÖRNER, 1939)

Genus: Xerobion NEVSKY, 1928

Species: judenkoi (SzELEGIEWICZ, 1959)

Tribe: Macrosiphini WILSON, $1910^{7}$

Genus: Acaudinum BÖRNER, 1930

Subgenus: Acaudinum BÖRNER, 1930

Species: centaureae (KoCH, 1854)

Genus: Acyrthosiphon MORDVILKO, 1914

Subgenus: Acyrthosiphon MORDVILKO, 1914

Species: auctum (WALKER, 1849)

boreale HILLE RIS LAMBERS, 1952

caraganae caraganae (CHOLODKOVSKY, 1907) !

cyparissiae (KOCH, 1855)

Subspecies: cyparissiae (KoCH, 1855) 
propinquum MORDVILKO, 1914

ignotum MORDVILKO, 1914

knechteli (BÖRNER, 1950)

lactucae (PASSERINI, 1860)

loti (THEOBALD, 1913)

malvae (MOSLEY, 1841)

Subspecies: agrimoniae (BÖRNER, 1940)

malvae (MOSLEY, 1841)

oterii PRIOR \& STROYAN, 1964

potha BÖRNER, 1950

rogersii (THEOBALD, 1913)

parvum BÖRNER, 1950

pisum HARRIS, 1776

Subspecies: ononis (KOCH, 1855)

pisum HARRIS, 1776

Subgenus: Liporrhinus BÖRNER, 1939

Species: chelidonii (KALTENBACH, 1843)

Genus: Allocotaphis BÖRNER, 1950

Species: quaestionis (BÖRNER, 1942)

Genus: Ammiaphis BÖRNER, 1952

Species: sii (KoCH, 1855)

Genus: Amphorophora BUCKTON, 1876

Subgenus: Amphorophora BUCKTON, 1876

Species: ampullata ampullata BUCKTON, 1876 gei (BÖRNER, 1939) idaei (BÖRNER, 1939) rubi (KALTENBACH, 1843)

Genus: Amphorosiphon HILLE RIS LAMBERS, 1949

Species: pulmonariae (BÖRNER, 1942)

Genus: Anuraphis DEL GUERCIO, 1907

Species: catonii HILLE RIS LAMBERS, 1935

farfarae (KoCH, 1854) subterranea (WALKER, 1852)

Genus: Aspidaphis GILLETTE, 1917

Species: adjuvans (WALKER, 1848)

Genus: Aulacorthum MORDVILKO, 1914

Subgenus: Aulacorthum MORDVILKO, 1914

Species: aegopodii BÖRNER, 1939 
cylactis BÖRNER, $1942^{8}$

flavum MÜLLER, 1958

knautiae HEIE, 1960

langei BÖRNER, 1939

majanthemi MÜLLER, 1956

palustre HILLE RIS LAMBERS, 1947

rufum HILLE RIS LAMBERS, 1947

sedens MÜLLER, 1966

solani solani (KALTENBACH, 1843)

speyeri BÖRNER, 1939

Genus: Brachycaudus VAN DER GOOT, 1913

Subgenus: Acaudus VAN DER GOOT, 1913

Species: divaricatae SHAPOSHNIKOV, 1956

klugkisti (BÖRNER, 1942)

lamii (KOCH, 1854)

lateralis WALKER, 1848

lucifugus MÜLLER, 1952

lychnidis (LINNAEUS, 1758)

mordvilkoi HILLE RIS LAMBERS, 1931

populi (DEL GUERCIO, 1911)

Subgenus: Appelia BÖRNER, 1930

Species: cerinthis BOZHKO, 1961

prunicola (KALTENBACH, 1943)

Subspecies: prunicola (KALTENBACH, 1943)

schwartzi (BÖRNER, 1931) ${ }^{9}$

Species: tragopogonis (KALTENBACH, 1843)

Subgenus Brachycaudina BÖRNER, 1930

Species: napelli (SCHRANK, 1801)

Subgenus: Brachycaudus VAN DER GOOT, 1913

Species: helichrysi (KALTENBACH, 1843)

salicinae BÖRNER, 1939

spiraeae BÖRNER, 1932

Subgenus: Prunaphis SHAPOSHNIKOV, 1964

Species: cardui (LINNAEUS, 1758) ${ }^{\mathbf{1 0}}$

Subgenus: Scrophulaphis ANDREEV, 1982

Species: linariae STROYAN, $1950{ }^{11}$

persicae (PASSERINI, 1860) 
Subgenus: Thuleaphis HILLE RIS LAMBERS, 1960

Species: amygdalinus (SCHOUTEDEN, 1905) rumexicolens (РATCH, 1917)! sedi (JАCOB, 1964)

Genus: Brachycolus BUCKTON, 1879

Species: cerastii (KALTENBACH, 1846) cucubali (PASSERINI, 1863) stellariae (HARDY, 1850)

Genus: Brachycorynella AIZENBERG, 1954 Species: asparagi (MORDVILKO, 1929)

Genus: Brevicoryne DAS, 1915

Species: brassicae (LINNAEUS, 1758)

Genus: Capitophorus VAN DER GOOT, 1913

Species: carduinus (WALKER, 1850) elaeagni (DEL GUERCIO, 1894) hippophaes hippophaes (WALKER, 1852) horni BÖRNER, 1931

Subspecies: gynoxantha HILLE RIS LAMBERS, 1953 horni BÖRNER, 1931 pakansus HOTTES \& FRISON, 1931 similis VAN DER GOOT, 1915

Genus: Cavariella Del GueRCIO, 1911

Species: aegopodii (SCOPOLI, 1763) archangelicae (SCOPOLI, 1763) cicutae (KоCH, 1854) intermedia HILLE RIS LAMBERS, 1969 konoi TAKAHASHI, 1939 pastinacae (LINNAEUS, 1758) salicicola (MATSUMURA, 1917) theobaldi (GILLETTE \& BRAGG, 1918)

Genus: Ceruraphis BÖRNER, 1926 Species: eriophori (WALKER, 1848) Genus: Chaetosiphon MORDVILKO, 1914

Subgenus: Chaetosiphon MORDVILKO, 1914 Species: alpestre alpestre HILLE RIS LAMBERS, 1953 Subgenus: Pentatrichopus BÖRNER, 1930

Species: potentillae (WALKER, 1850) tetrarhodum (WALKER, 1849) 
Genus: Chondrillobium BOZHKO, 1961

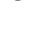

444

445

446

447

448

449

450

451

452

453

454

455
Species: blattnyi (PINTERA, 1959)

Genus: Coloradoa WILSON, 1910

Species: abrotani (KoCH, 1854) absinthii (LICHTENSTEIN, 1885)

achilleae HILLE RIS LAMBERS, 1939

angelicae (DEL GUERCIO, 1911)

artemisiae (DEL GUERCIO, 1913)

campestrella OSSIANNILSSON, 1959

campestris BÖRNER, 1939

huculaki SZELEGIEWICZ, $1981^{\mathbf{1 2}}$

inodorella OSSIANNILSSON, 1959

tanacetina (WALKER, 1850)

Genus: Corylobium MORDVILKO, 1914

Species: avellanae (SCHRANK, 1801)

Genus: Cryptaphis HILLE RIS LAMBERS, 1947

Species: poae (HARDY, 1850)

Genus: Cryptomyzus OESTLUND, 1923

Subgenus: Ampullosiphon HEIKINHEIMO, 1955

Species: stachydis (HEIKINHEIMO, 1955)

Subgenus: Cryptomyzus OESTLUND, 1922

Species: alboapicalis (THEOBALD, 1916)

ballotae HILLE RIS LAMBERS, 1953

galeopsidis (KALTENBACH, 1843)

Subspecies: citrinus HILLE RIS LAMBERS, 1953

dickeri HILLE RIS LAMBERS, 1953

galeopsidis (KALTENBACH, 1843)

korschelti BÖRNER, 1938

leonuri BOZHKO, 1961

ribis (LINNAEUS, 1758)

Genus: Cryptosiphum BUCKTON, 1879

Species: artemisiae artemisiae BUCKTON, 1879

brevipilosum BÖRNER, 1932

Genus: Delphiniobium MORDVILKO, 1914

Species: junackianum junackianum (KARSCH, 1887)

Genus: Diphorodon BÖRNER, $1939^{13}$

Species: cannabis PASSERINI, 1860 
Genus: Diuraphis AIZENBERG, 1956

Subgenus: Diuraphis AIZENBERG, 1956

467

468

469

471

472

473

474

475

476

477

478

479

480

481

482

483

484

485

486

487

488

489

490

491

492

493

494
Species: muehlei (BÖRNER, 1950)

noxia (MORDVILKO ex KURDJUMOV, 1913)

Subgenus: Holcaphis HILLE RIS LAMBERS, 1939

Species: agrostidis (MUDDATHIR, 1965)

bromicola (HILLE RIS LAMBERS, 1959)

calamagrostis (OSSIANNILSSON, 1959)

frequens (WALKER, 1848)

holci (HILlE RIS LAMBERS, 1956)

\section{Genus: Dysaphis BöRNER, 1931}

Subgenus: Dysaphis BÖRNER, 1931

Species: angelicae (KOCH, 1854)

anthrisci BÖRNER, 1950

apifolia (THEOBALD, 1923)

Subspecies: apiifolia petroselini (BÖRNER, 1950)

bonomii (HILLE RIS LAMBERS, 1935)

brancoi brancoi (BÖRNER, 1950)

chaerophylli (BÖRNER, 1940)

crataegi (KALTENBACH, 1843)

Subspecies: aethusae (BÖRNER, 1950)

crataegi (KALTENBACH, 1843)

kunzei (BÖRNER, 1950)

devecta (WALKER, 1849)

hirsutissima (BÖRNER, 1940)

lappae (KOCH, 1854)

Subspecies: cirsii (BÖRNER, 1950)

lappae (KOCH, 1854)

laserpitii (BÖRNER, 1950)

lauberti (BÖRNER, 1940)

leefmansi (HILLE RIS LAMBERS, 1954)

Subspecies: leefmansi krumbholzi MÜLLER, 1961

newskyi (BÖRNER, 1940)

Subspecies: aizenbergi (SHAPOSHNIKOV, 1949)

newskyi (BÖRNER, 1940)

oreoselini SZELEGIEWICZ, 1982

radicola (MORDVILKO, 1897)

ranunculi (KALTENBACH, 1843) 
tulipae (BOYER DE FONSCOLOMBE, 1841)

496

497

498

499

500

501

502

503

504

505

506

507

508

509

510

511

512

513

514

515

516

517

518

519

520

521
Subgenus: Pomaphis BÖRNER, 1939

Species: aucupariae (BUCKTON, 1879)

plantaginea (PASSERINI, 1860)

plantaginis (PAŠEK, 1955)

pyri (BOYER DE FONSCOLOMBE, 1841)

sorbi (KALTENBACH, 1843)

Genus: Elatobium MORDVILKO, 1914

Species: abietinum (WALKER, 1849)

Genus: Ericaphis BÖRNER, 1939

Species: ericae (BÖRNER, 1933) latifrons (BÖRNER, 1942)

Genus: Hayhurstia DEL GUERCIO, 1917

Species: atriplicis (LINNAEUS, 1761)

Genus: Hyadaphis KIRKALDY, 1904

Species: bicincta BÖRNER, 1942

bupleuri BÖRNER, 1939

coriandri (DAS, 1918)

foeniculi foeniculi (PASSERINI, 1860)

passerinii (DEL GUERCIO, 1911)

polonica SZELEGIEWICZ, 1959

tataricae (AIZENBERG, 1935)

Genus: Hyalopteroides THEOBALD, 1916

Species: humilis (WALKER, 1852)

Genus: Hydaphias BÖRNER, 1930

Species: hofmanni BÖRNER, 1950

molluginis BÖRNER, 1939

mosana HILLE RIS LAMBERS, 1956

Genus: Hyperomyzus BÖRNER, 1933

Subgenus: Hyperomyzella HILLE RIS LAMBERS, 1949

Species: rhinanthi (SCHOUTEDEN, 1903)

Subgenus: Hyperomyzus BÖRNER, 1933

Species: lactucae (LINNAEUS, 1758)

lampsanae (BÖRNER, 1932)

pallidus HILLE RIS LAMBERS, 1935

Subgenus: Neonasonovia HILLE RIS LAMBERS, 1949

Species: picridis (BÖRNER \& BLUNCK, 1916)

zirnitsi HILLE RIS LAMBERS, 1952 
Genus: Idiopterus DAVIS, 1909

Species: nephrelepidis DAVIS, 1909** !

Genus: Illinoia WILSON, 1910

Subgenus: Illinoia WILSON, 1910

Species: azaleae azaleae (MASON, 1925) !

Subgenus Masonaphis HILLE RIS LAMBERS, 1939

Species: lambersi (MACGILLIVRAY, 1960) !

Genus: Impatientinum MORDVILKO, 1914

Species: asiaticum asiaticum NEVSKY, 1929 ! balsamines (KALTENBACH, 1862)

Genus: Jacksonia THEOBALD, 1923

Species: papillata THEOBALD, 1923

Genus: Linosiphon BÖRNER, 1950

Species: asperulophagum HOLMAN, 1961 galii (MAMONTOVA, 1961) galiophagum (WIMSHURST, 1923)

Genus: Liosomaphis WALKER, 1868

Species: berberidis (KALTENBACH, 1843)

Genus: Lipaphis MORDVILKO, 1928

Subgenus: Lipaphidiella DONCASTER, 1954

Species: ruderalis BÖRNER, 1939

Subgenus: Lipaphis MORDVILKO, 1928

Species: alliariae F. P MÜLLER, 1952 erysimi (KALTENBACH, 1843) fritzmuelleri BÖRNER, 1950 rossi rossi BÖRNER, 1939 turritella (WAHLGREN, 1938)

Genus: Longicaudus VAN DER GOOT, 1913

Species: trirhodus trirhodus (WALKER, 1849)

Genus: Macrosiphoniella DEL GUERCIO, 1911

Subgenus: Asterobium HILLE RIS LAMBERS, 1938

Species: asteris (WALKER, 1849) linariae (KоCH, 1855)

Subgenus: Macrosiphoniella DEL GUERCIO, 1911

Species: abrotani abrotani (WALKER, 1852) absinthii (LINNAEUS, 1758) artemisiae artemisiae (BOYER DE FONSCOLOMBE, 1841) 
dimidiata BÖRNER, 1942

kaufmanni BÖRNER, 1940

millefolii (DE GEER, 1773)

nitida nitida (BÖRNER, 1950)

ptarmicae HILLE RIS LAMBERS, 1956

sanborni (GILLETTE, 1908) ** !

sejuncta (WALKER, 1848)

staegeri staegeri HILLE RIS LAMBERS, 1947

subaequalis BÖRNER, 1942

subterranea (KOCH, 1855)

tanacetaria tanacetaria (KALTENBACH, 1843)

tapuskae (HOTTES \& FRISON, 1931)

usquertensis HILLE RIS LAMBERS, 1935

Subgenus: Phalangomyzus BÖRNER, 1939

Species: oblonga (MORDVILKO, 1901

persequens (WALKER, 1852)

Subgenus: Ramitrichophorus HILLE RIS LAMBERS, 1947

Species: hillerislambersi OSSIANNILSSON, 1954

jankei BÖRNER, 1939

medvedevi (BOZHKO, 1957)

Genus: Macrosiphum PASSERINI, 1860

Subgenus: Macrosiphum PASSERINI, 1860

Species: albifrons ESSIG, 1911 !

atragenae HOLMAN, 1980

cholodkovskyi (MORDVILKO, 1909)

daphnidis BÖRNER, 1950

dryopteridis (HOLMAN, 1959) ${ }^{\mathbf{1 4}}$

equiseti (HOLMAN, 1961) ${ }^{\mathbf{1 5}}$

euphorbiae (THOMAS, 1878) !

euphorbiellum THEOBALD, $1917^{16}$

funestum (MACCHIATI, 1885)

gei (KOCH, 1855)

holmani LECLANT, 1972

knautiae HOLMAN, 1972

lisae HEIE, 1965

melampyri MORDVILKO, 1919

oredonense REMAUDIÉRE, 1952

prenanthidis BÖRNER, 1940 
ptericolens PATCH, $1919{ }^{17}$ !

rosae rosae (LINNAEUS, 1758)

stellariae THEOBALD, 1913

tinctum (WALKER, 1849)

Genus: Megoura BUCKTON, 1876

Species: litoralis F. P. MÜLLER, 1952 viciae BUCKTON, 1876

Genus: Megourella HILLE RIS LAMBERS, 1949

Species: purpurea HILLE RIS LAMBERS, 1949 tribulis (WALKER, 1849)

Genus: Metopeurum MORDVILKO, 1914

Species: enslini (BÖRNER, 1933) fuscoviride fuscoviride STROYAN, 1950

Genus: Metopolophium MORDVILKO, 1914

Subgenus: Metopolophium MORDVILKO, 1914

Species: albidum HILLE RIS LAMBERS, 1947 dirhodum (WALKER, 1849) fasciatum STROYAN, 1982 festucae festucae (THEOBALD, 1917) tenerum HILLE RIS LAMBERS, 1947

Genus: Microlophium MORDVILKO, 1914

Species: carnosum (BUCKTON, 1876) sibiricum sibiricum (MORDVILKO, 1914)

Genus: Microsiphum CHOLODKOVsKY, 1902

Species: jazykovi NEVSKY, 1928 millefolii WAHLGREN, 1940 woronieckae JUDENKO, 1931

Genus: Muscaphis BÖRNER, 1933

Species: escherichi escherichi (BÖRNER, 1939) musci BÖRNER, 1933

Genus: Myzaphis VAN DER GOOT, 1913

Species: rosarum (KALTENBACH, 1843)

Genus: Myzus PASSERINI, 1860

Subgenus: Galiobium BÖRNER, 1933

Species: langei (BÖRNER, 1933)

Subgenus: Myzus PASSERINI, 1860

Species: cerasi

Subspecies: cerasi (FABRICIUS, 1775) 
601

602

603

604

605

606

607

608

609

610

612

613

614

615

616

617

618

619

620

621

622

623 pruniavium BÖRNER, 1926

lythri (SCHRANK, 1801)

ornatus LAING, 1932 !

varians DAVIDSON, 1912 !

Subgenus: Nectarosiphon SCHOUTEDEN, 1901

Species: ajugae SCHOUTEDEN, 1903

certus (WALKER, 1849)

ligustri (MOSLEY, 1841)

myosotidis (BÖRNER, 1950)

persicae (SULZER, 1776) !

titschaki (BÖRNER, 1942)

Subgenus: Sciamyzus STROYAN, 1954

Species: ascalonicus DONCASTER, 1946 !

Genus: Nasonovia MoRdVILKO, 1914

Subgenus: Nasonovia MORDVILKO, 1914

Species: compositellae (THEOBALD, 1924)

Subspecies: compositellae (THEOBALD, 1924)

nigra (HILLE RIS LAMBERS, 1931)

pilosellae (BÖRNER, 1933)

ribisnigri (MOSLEY, 1841)

Genus: Neoamphorophora MASON, 1924

Species: ledi (WAHLGREN, 1938)

Genus: Neomariaella SZWEDO \& OSIADACZ, 2010

Species: lambersi (SZELEGIEWICZ, 1961)

Genus: Neomyzus VAN DER GOOT, 1915

Species: circumflexus (BUCKTON, 1876) !

Genus: Neopterocomma HILLE RIS LAMBERS, 1935

Species: asiphum HILLE RIS LAMBERS, 1935 verhoeveni HILLE RIS LAMBERS, 1956

Genus: Ossiannilssonia HILLE RIS LAMBERS, 1952

Species: oelandica HILLE RIS LAMBERS, 1952

Genus: Ovatomyzus HILLE RIS LAMBERS, 1947

Species: boraginacearum EASTOP, 1952

chamaedrys (PASSERINI, 1879)

stachyos HILLE RIS LAMBERS, 1947

Genus: Ovatus VAN DER GOOT, 1913

Subgenus: Ovatus VAN DER GOOT, 1913

Species: crataegarius (WALKER, 1850) 
625

626

627

628

629

630

631

632

633

634

635

636

637

638

639

640

641

642

643

644

645

646

647

648

649

650

651 insitus (WALKER, 1849)

mentharius (VAN DER GOOT, 1913)

Genus: Paczoskia MORDVILKO, 1919

Species: longipes TASHEV, 1964

major major BÖRNER, 1950

obtecta BÖRNER, 1950

Genus: Paramyzus BÖRNER, 1933

Species: heraclei heraclei BÖRNER, 1933

Genus: Phorodon PASSERINI, 1860

Species: humuli humuli (SCHRANK, 1801)

Genus: Pleotrichophorus BÖRNER, 1930

Species: deviatus MÜLLER, 1972

duponti HILLE RIS LAMBERS, 1935

glandulosus glandulosus (KALTENBACH, 1846)

helichrysi BOZHKO, 1963

persimilis persimilis BÖRNER, 1950

Genus: Plocamaphis OESTLUND, 1923

Species: amerinae (HARTIG, 1841)

flocculosa (WEED, 1891)

Subspecies: flocculosa brachysiphon OSSIANNILSSON, 1959

flocculosa goernitzi BÖRNER, 1940

Genus: Pseudacaudella BÖRNER, 1950

Species: rubida (BÖRNER, 1939)

Genus: Pseudobrevicoryne HEINZE, 1960

Species: buhri (BÖRNER, 1952)

erysimi HOLMAN, 1961

Genus: Pterocomma BUCKTON, 1879

Species: dubium (BÖRNER, 1950)

jacksoni THEOBALD, 1921

konoi (HORI ex TAKAHASHI, 1939)

pilosum BUCKTON, 1879

Subspecies: pilosum BUCKTON, 1879

sarmaticum SZELEGIEWICZ, 1967

populeum (KALTENBACH, 1843)

rufipes (HARTIG, 1841)

salicis (LINNAEUS, 1758)

tremulae BÖRNER, 1940 
Genus: Rhodobium HILLE RIS LAMBERS, 1947

Genus: Rhopalomyzus MORDVILKO, 1921

Subgenus: Judenkoa HILLE RIS LAMBERS, 1946

Species: poae (GILLETTE, 1908)

Genus: Rhopalosiphoninus BACKER (A. C.), 1920

Subgenus: Neorhopalosiphoninus GHOSH (A.K.)

$$
\text { \& RAYCHAUDHURI (D.N.), } 1968^{\mathbf{1 8}}
$$

Species: staphyleae (KOCH, 1854)

Subspecies: staphyleae (KoCH, 1854)

Subgenus: Pseudorhopalosiphoninus HEINZE, 1961

Species: calthae (KоCH, 1854)

Subgenus: Rhopalosiphoninus BACKER, 1920

Species: latysiphon (DAVIDSON, 1912) ! maianthemi STROYAN, 1965 ribesinus (VAN DER GOOT, 1912)

Subgenus: Submegoura HILLE RIS LAMBERS, 1953

Species: heikinheimoi (BÖRNER, 1952)

Genus: Semiaphis VAN DER GOOT, 1913

Species: anthrisci (KALTENBACH, 1843) dauci dauci (FABRICIUS, 1775) pimpinellae (KALTENBACH, 1843)

Genus: Sitobion MORDVILKO, 1914

Subgenus: Sitobion MORDVILKO, 1914 avenae (FABRICIUS, 1775) fragariae (WALKER, 1848) paludum MÜLLER, 1982

Genus: Smiela MORDVILKO, 1948 
690

691

692

693

694

695

696

697

698
Genus: Subacyrthosiphon HILLE RIS LAMBERS, 1947

Species: cryptobium HILLE RIS LAMBERS, 1947

Genus: Taiwanomyzus TAO, 1963

Species: alpicola (HILLE RIS LAMBERS, 1969)

Genus: Titanosiphon NEVSKY, 1928

Species: artemisiae (КоCH, 1855)

Genus: Trichosiphonaphis TAKAHASHI, 1922

Subgenus: Xenomyzus AIZENBERG, 1935

Species: corticis (AIZENBERG, 1935)

Genus: Tubaphis HILLE RIS LAMBERS, 1947

Species: ranunculina (WALKER, 1852)

Genus: Uroleucon MORDVILKO, 1914

Subgenus: Lambersius OLIVE, 1965

Species: erigeronense (THOMAS, 1878) !

Subgenus: Uroleucon MORDVILKO, 1914

Species: achilleae (KocH, 1855)

chondrillae (NEVSKY, 1929)

cichorii (КоCH, 1855)

cirsii (LINNAEUS, 1758)

grossum (HILLE RIS LAMBERS, 1939)

hypochoeridis (FABRICIUS, 1779)

inulicola inulicola (HILLE RIS LAMBERS, 1939)

jaceicola jaceicola (HILLE RIS LAMBERS, 1939)

leontodontis (HILLE RIS LAMBERS, 1939)

murale (BUCKTON, 1876)

obscurum (КосH, 1855)

picridis (FABRICIUS, 1775)

pilosellae (BÖRNEr, 1933)

pseudoambrosiae (OLIVE, 1963) ** !

pulicariae (HILle RIS LAMBERS, 1939)

sonchi sonchi (LINNAEUS, 1767)

tanaceti (LINNAEUS, 1758)

telekiae (HOLMAN, 1965)

tussilaginis (WALKER, 1850)

Subgenus: Uromelan MORDVILKO, 1914

Species: aeneum (HILLE RIS LAMBERS, 1939)

campanulae campanulae (KALTENBACH, 1843)

carlinae (BÖRNER, 1933) 
699

700

701

702

703

704

705

706

707

708

709

710

711

712

714

715

716

717

718

719

720

721

722

723

724

725

726 doronici (BÖRNER, 1942)

ensifoliae (HOLMAN, 1965)

jaceae (LINNAEUS, 1758)

Subspecies: jaceae (LINNAEUS, 1758)

henrichi (BÖRNER, 1950)

reticulatum (HILLE RIS LAMBERS, 1939)

minosmartellii BARBAGALLO \& PATTI, 1994

nigrocampanulae (THEOBALD, 1928)

rapunculoidis (BÖRNER, 1939)

simile (HILLE RIS LAMBERS, 1935)

solidaginis (FABRICIUS, 1779)

taraxaci (KALTENBACH, 1843)

Genus: Vesiculaphis DEL GUERCIO, 1911

Species: theobaldi TAKAHASHI, 1930

Genus: Volutaphis BÖRNER, 1939

Species: schusteri (BÖRNER, 1939)

Genus: Wahlgreniella HILLE RIS LAMBERS, 1949

Species: ossiannilssoni HILLE RIS LAMBERS, 1949

Genus: Zinia SHAPOSHNIKOV, 1950

Species: veronicae SHAPOSHNIKOV, 1950

Subfamily: LACHNINAE HERRICH-SCHAEFFER, 1854

Tribe: Eulachnini BAKER, 1920

Genus: Cinara CURTIS, 1835

Subgenus: Cinara CURTIS, 1835

Species: acutirostris HILLE RIS LAMBERS, 1956

brauni BÖRNER, 1940

cembrae (SEITNER, 1936)

confinis (KOCH, 1856)

costata (ZETTERSTEDT, 1828)

cuneomaculata (DEL GUERCIO, 1909)

hyperophila (KoCH, 1855)

kochiana kochiana (BÖRNER, 1939)

laricis (HARTIG, 1839)

neubergi (ARNHART, 1930)

nuda MORDVILKO, 1895

pectinatae (NÖRDLINGER, 1880)

piceae (PANZER, 1800) 
piceicola (CHOLODKOVSKY, 1896)

pilicornis (HARTIG, 1841)

pilosa (ZETTERSTEDT, 1940)

pinea (MORDVILKO, 1895)

pini (LINNAEUs, 1758)

pinihabitans (MORDVILKO, 1895)

piniphila (RATZEBURG, 1844)

pruinosa pruinosa (HARTIG, 1841)

Subgenus: Cupressobium BÖRNER, 1940

Species: cupressi (BUCKTON, 1881)

fresai (BLANCHARD, 1939)

juniperi (DE GEER, 1773)

mordvilkoi (PAŠEK, 1954)

tujafilina (DEL GUERCIO, 1909)

Genus: Eulachnus Del GUERCIO, 1909

Species: agilis (KALTENBACH, 1843)

alticola BÖRNER, 1940

brevipilosus BÖRNER, 1940

cembrae BÖRNER, 1950

nigricola (PAŠEK, 1953)

rileyi (WILLIAMS, 1911)

Genus: Schizolachnus MORDVILKO, 1909

Species: pineti (FABRICIUS, 1781)

Tribe: Lachnini HERRICH-SCHAEFFER, 1854

Genus: Lachnus BURMEISTER, 1835

Species: pallipes (HARTIG, 1841) ${ }^{20}$

roboris (LINNAEUS, 1758)

Genus: Maculolachnus GAUMONT, 1920

Species: submacula (WALKER, 1848)

Genus: Stomaphis WALKER, $1870^{21}$

Subgenus: Parastomaphis PAŠEK, 1953

Species: graffii CHOLODKOVSKY, 1894

longirostris (FABRICIUS, 1787)

Subgenus: Stomaphis WALKER, 1870

Species: quercus (LINNAEUS, 1758)

wojciechowskii DEPA, 2012 
Genus: Tuberolachnus MORDVILKO, 1909

Subgenus: Tuberolachnus MORDVILKO, 1909

754

Species: salignus (GMELIN, 1790)

Tribe: Tramini HERRICH-SCHAEFFER, 1854

Genus: Protrama BAKER (A.C.), 1920

Species: flavescens (KoCH, 1856) ranunculi (DEL GUERCIO, 1909)

Genus: Trama von Heyden, 1837

Subgenus: Neotrama BAKER (A.C.), 1920 maritima (EASTOP, 1953)

Subgenus: Trama von HEYDEN, 1837

Species: centaureae BÖRNER, 1940 rara MORDVILKO, 1908 troglodytes VON HEYDEN, 1837

\section{Taxonomix comments}

${ }^{1}$ According to BLACKMAN \& EASTOP (2015) Daktulosphaira SHIMER, $1866=$ Viteus SHIMER, 1867.

${ }^{2}$ This species was included in the systematic reviews of Polish aphids by SZELEGIEwICZ (1978a), NAST et al. (1990), WEGIEREK \& WOJCIECHOWSKI (2004) and OSIADACZ \& HAŁAJ (2009, 2010) without any distributional data; its presence has now been confirmed: Katowice, Acer pseudoplatanus LINNAEUS, 173, 16.VI.2013, No 15/13/121, UŚ, leg. K. WIECZOREK.

3 According to García Prieto et al. (2004): "Hille Ris Lambers (1966, pg. 125) established Aphis davletshinae to replace A. althaeae (NEvSKY, 1929) because he believed the latter to be a senior homonym of althaea HARRIS, 1776 (nomen dubium according to EASTOP \& Hille Ris LAMBERS, 1976, pg. 42). However, this was a mistake, opposed to what is stated in article 58.1 of the International Code of Zoological Nomenclature, Fourth Edition" (CINZ 2000). A. davletshinae should be A. althaeae.

${ }^{4}$ According to GARCÍA PRIETO et al. (2004): "For two aphid species in the subfamily Aphidinae living on Ballota (one belonging to the genus Aphis and the other to Brachycaudus) SZELEGIEwICZ (1968) assigned the name Aphis ballotae to the latter and established the replacement name balloticola for the former. This was not the correct 
choice as there are two specimens seen by PASSERINI (much later designated as lectotypes by HiLle RIS LAMBERS of A. ballotae PASSERINI, unpublished datum!), currently at the Natural History Museum - London, belonging to the group frangulaelgossypii of the genus Aphis (BLACKMAN, pers. comm., 2004)".

Also, Aphis balloticola SZELEGIEWICZ, 1968 is a nomen nudum because when it was established as a new name for «Aphis ballotae: auct., non PASSERINI, 1860», SZELEGIEWICZ gave no description or indication: the bibliographic reference to SZULCZEWSKI is not sufficient as he (SZULCZEWSKI, 1950, pg. 34) did not provide any characters for his "Aphididae 34". A. balloticola SZELEGIEWICZ should be A. ballotae PASSERINI and the former name has been excluded from the checklist.

5 According to García PRIETO et al. (2004): "SZELEGIEWICZ (1961, pg. 303) established Aphis stroyani to replace A. picridis (BÖRNER, 1950) (junior secondary homonym of A. picridis FABRICIUS, 1775). However, this was a mistake, contrary to what is stated for the replacement of junior secondary homonyms in article 59.4 of the International Code of Zoological Nomenclature, Fourth Edition" (CINZ 2000). A. stroyani SZELEGIEWICZ should be A. picridis (BÖRNER).

${ }^{6}$ The correct name for the rice root aphid is Rhopalosiphum rufiabdominale (SASAKI), not rufiabdominalis (mentioned by ŁABANOWSKI (2008) and OSIADACZ \& HAŁAJ (2012)), because the gender of Rhopalosiphum is neuter (BLACKMAN \& EASTOP 2011).

${ }^{7}$ All former Pterocommatinae have been included in the tribe Macrosiphini WILSON, 1910 in accordance with VON DOHLEN et al. (2006). Not confirmed by NIETO NAFRÍA \& FAVRET (2011) - the Pterocommatinae has the status of an independent subfamily; however, the authors in the update to the registers of family-group and genus group taxa of Aphidoidea (2014) included all former Pterocommatinae in Macrosiphini.

${ }^{8}$ Aulacorthum cylactis BÖRNER, 1942 - according to BLACKMAN \& EASTOP (2015) this is a good species, not the subspecies Aulacorthum (Aulacorthum) solani cylactis BÖRNER, 1942.

${ }^{9}$ A morphometric and molecular study by CocuzZA et al. (2007) indicated that this species should be treated as a subspecies of $B$. (A.) prunicola.

${ }^{10}$ According to the subgeneric classification by ANDREEV (2003) Brachycaudus cardui (LINNAEUS, 1758) belongs to the subgenus Prunaphis.

11 According to the subgeneric classification by ANDREEV (2003) Brachycaudus linariae STROYAN, 1950 belongs to the subgenus Scrophulaphis.

12 This species was included in the systematic reviews of Polish aphids by SZELEGIEWICZ (1978a), NAST et al. (1990), WEGIEREK \& WOJCIECHOWSKI (2004) and OSIADACZ \& HAEAJ $(2009,2010)$ without any distribution data; its presence has now been confirmed. The locality of Coloradoa huculaki (Poland, Warszawa-Bielany, 27.06.1976, 
SzELEGIEWICZ 1981, p. 85) is mentioned in "The Aphids (Homoptera, Aphidoidea) of the Hortobagy" (SZELEGIEWICZ 1981).

${ }^{13}$ NIETO NAFrí́A \& FAVRET 2011 gave full generic status to Diphorodon BöRNER, 1939.

${ }^{14}$ According to JENSEN (1997) Sitobion (S.) dryopteridis (HOLMAN, 1959), mentioned in OSIADACZ \& HALAJ 2010, should be Macrosiphum (M). dryopteridis (HOLMAN, 1959).

${ }^{15}$ According to JeNSEN (1997) Sitobion (S.) equiseti (Holman, 1961), mentioned in OSIADACZ \& HAŁAJ 2010, should be Macrosiphum (M.) equiseti (Holman, 1961).

${ }^{16}$ Macrosiphum euphorbiellum THEOBALD, 1917 (= M. amygdaloides THEOBALD, 1925). Synonymy established by WATSON (1982) (PhD thesis) and published in BLACKMAN 2010.

${ }^{17}$ According to JENSEN (1997) Sitobion (S.) ptericolens (РATCH, 1919), mentioned in OSIADACZ \& HAŁAJ 2010, should be Macrosiphum (M.) ptericolens PATCH, 1919.

${ }^{18}$ According to NiETO NAFRÍA et al. (2009): "HILLE RIS LAMBERS never described the genus Myzosiphon, but EASTOP \& HILLE RIS LAMBERS (1976: 378) used it as a subgenus of Rhopalosiphoninus BAKER, also failing to describe it, and it has continued to be used as such. As Myzosiphon is unavailable and cannot be valid (Code article 23.1), it requires a replacement name. The replacement name must be Neorhopalosiphoninus GHOSH \& RAYCHAUDHURI (1968: 187), because it is the only available synonym".

${ }^{19}$ Krośnice, 27.VI.2013, No 06/13/135, UŚ, leg. Ł. DEPA.

${ }^{20}$ A morphometric and molecular study by MRÓZ et al. 2015 indicated that $L$. longirostris (MORDVILKO, 1909) is a synonym of L. pallipes (HARTIG, 1841).

${ }^{21}$ Parastomaphis PAŠEK, 1953 stat. rev. (DEPA et al. 2015). On the basis of the structure of the apical part of antennomere VI (rounded apex in Stomaphis and nodulose apex in Parastomaphis) of the viviparous generation, PAŠEK (1953) subdivided the genus Stomaphis into two subgenera: Stomaphis and Parastomaphis, later treated as separate genera (SZELEGIEWICZ 1978b). Then Parastomaphis was synonymised with Stomaphis by EASTOP \& HILLE RIS LAMBERS (1976) and current aphid taxonomy retains this taxon undivided as a single genus within the tribe Lachnini (NIETO NAFRIA \& FAVRET 2011). However, on the basis of the morphological characters of the sexuales, supported by biological data, in our opinion the taxonomic status of the subgenus Parastomaphis should be maintained.

\section{ACKNOWLEDGEMENTS}

The authors are sincerely grateful to STEPHANIE WRIGHT (BMNH, London, UK) for her linguistic assistance. We are also very grateful to the anonymous Reviewers and the Editor for all the valuable suggestions that have improved the manuscript. 


\section{REFERENCES}

ANDREEV A. 2003. The subgeneric classification of Brachycaudus VAN DER GOOT. [in:] J.C. Simon, C.A. Dedryver, C. Rispe, M. Hulle (eds.). Aphids in a New Millenium, Inra Editions, Rennes, 111-117.

BLACKMAN R.L. 2010. Handbooks for the identification of British insects 2, 7. Aphids Aphidinae (Macrosiphini). Royal Entomological Society, London.

BlaCKMAN R.L., EASTOP V.F. 1994. Aphids on Natural History Museum, Wallingford.

BLACKMAN R.L., EASTOP V.F. 2011. Additions and amendments to "Aphids on the World's Plants". Zootaxa 2774: 57-68.

BLACKMAN R.L., EASTOP V.F. 2015. Aphids of the World's Plants: An Online Identification and Information Guide. Internet: http://www.aphidsonworldsplants.info (Accessed 8 Jun. 2015)

Cocuzza G., Cavalieri V., Jousselin E., Coeur D’ACier A., Barbagallo S. 2007. Morphological and molecular analysis of Brachycaudus, subgenus Appelia-complex (Hemiptera: Aphididae). Redia 90: 33-49.

Coeur D’acier A., Pérez Hidalgo N., Petrović-Obradović O. 2010. Chapter 9.2. Aphids (Hemiptera, Aphididae). [in]: A. Roques, M. KenIS, D. LeEs, C. LoPeZVAAMONDE, W. RABITSCH, J.-Y. RASPLUS, D.B. ROY (eds.). Alien terrestrial arthropods of Europe. BioRisk 4(1): 435-474.

CINZ [Comisión Internacional de Nomenclatura Zoológica] 2000. Código Internacional de Nomenclatura Zoológica Cuarta edición. Museo Nacional de Cencias Naturales (CSIC), Madrid.

DEPA Ł., KANTURSKI M., JUNKIERT Ł., WIECZOREK K. 2015. Giant females vs dwarfish males of the genus Stomaphis WALKER (Hemiptera: Aphididae) - an aphid example of the ongoing course to permanent parthenogenesis? Arthropod Systematics and Phylogeny 73(1): 19-40.

EAStop V.F., Hille Ris Lambers D. 1976. Survey of the World's Aphids. W. Junk, The Hague.

García Prieto F., Pérez Hidalgo N., Mier Durante M,P., Nieto Nafría J.M. 2004. Updated check-list of Iberian-Balearic Aphidini (Hemiptera, Aphididae). Graellsia 60(2): 197-214.

GHOSH A.K., RAYCHAUDHURI D.N. 1968. Aphids of Sikkim. Proceedings of the Zoological Society of Calcutta 21: 179-195.

HILLE RIS LAMBERS D. 1966. Some synonyms in Aphididae. Entomologische Berichten 26: $124-126$.

JENSEN A.S. 1997. Redefinition of the aphid genus Sitobion MoRDVILKO (Hemiptera: Aphididae) based on cladistics analyses, with emphasis on North American species. Systematic Entomology 22(4): 333-344. 
MrÓz E., TREla J., DePA Ł. 2015. Taxonomic analysis of Lachnus pallipes/longirostrisroboris complex (Hemiptera, Aphididae, Lachninae), with the redescription of sexual morphs and new synonymy. Zoologischer Anzeiger 254: 51-61.

NAst J., Chudzicka E., KlimaszewsKi S.M., CZYloK A., WoJCIECHOWSKI W., Koteja J. 1990. Homoptera - pluskwiaki równoskrzydłe. [in:] J. RAZOWSKI (ed.). Tom I., część XXXII/1 20. Ossolineum, Wrocław - Warszawa - Kraków: 91-124.

Nieto Nafría J.M., FAvret C., AKimoto S.-I., Barbagallo S., Chakrabarti S., Mier Durante M.P., Miller G.L., Pérez Hidalgo N., QiaO G-X., Sano M., STEKOLSHCHIKOV A., WEGIEREK P. 2009. Several Nomenclatural clarifications on genus-group names in the Aphididae (Hemiptera, Sternorrhyncha). Redia 92:119-123.

Nieto Nafría J.M., Favret C., AKimoto S.-I., Barbagallo S., Chakrabarti S., Mier Durante M.P., Miller G.L., Pérez Hidalgo N., QiaO G-X., SANO M., STEKOLSHCHIKOV A., WEGIEREK P. 2011. Register of genus-group taxa of Aphidoidea. [in:] J.M. NIETO NAFRÍA, C. FAVRET (eds.). Registers of Family-group and genus-group taxa of Aphidoidea (Hemiptera Sternorrhyncha). Universitad de León, León, 81-404.

NIETO NAFRÍA J.M., FAVRET C. 2014. Update to the Registers of family-group and genus group taxa of Aphidoidea (Hemiptera, Sternorrhyncha). Boletín de la Asociación Española de Entomología 38(1-2): 1-23.

Osiadacz B., HaŁaj R. 2009. Polish Entomological Monographs, vol. 6. The Aphids (Hemiptera: Sternorrhyncha: Aphidinea) of Poland. A distributional checklist. Polish Entomological Society, Poznań.

OsiadACZ B., HaŁAJ R. 2010. Silesian Natural History Monographs, vol. 1. Systematic Review of Aphids (Hemiptera: Sternorrhyncha: Aphidomorpha) of Poland with Host Plant Index. A Monographic Survey. Instytut Tarnogórski i Muzeum, Tarnowskie Góry.

OSIADACZ B., HAŁAJ R. 2011. Alien and invasive species of oviparous aphids (Aphidomorpha: Adelgoidea, Phylloxeroidea) in Poland: characteristics and review. Aphids and Other Hemipterous Insects 17: 31-40.

OSIADACZ B., HAŁAJ R. 2012. The Update of "Systematic Review of Aphids (Hemiptera: Sternorrhyncha: Aphidomorpha) of Poland with Host Plant Index". Wiadomości Entomologiczne 31(4): 230-241.

PAŠEK V. 1953. Contribution to the classification of Middle European Lachnidae (Homopt., Aphidoidea). Věstník Československé Zoologické Společnosti 17(3): 149-176. (in Czech)

SOIKA G., ŁABANOWSKI G. 2014. Invasive organisms recorded in Polish nursery gardens. Manual of detection of invasive mites and insects on the basis of their appearance and diagnostic features. Instytut Ogrodnictwa, Skierniewice. (in Polish)

SZELEGIEWICZ H. 1961. Über zwei wenig bekannte mitteleuropäische Blattlausarten (Homoptera: Aphididae). Bulletin de l'Académie Polonaise des Sciences (II) 9(7): 303-307.

SzELEGIEwICZ H. 1968. Aphids. Aphidodea. Catalogus faunae Poloniae 21(4): 1-316. (in Polish)

SZELEGIEWICZ H. 1978a. A check list of of the aphids of Poland. Zeszyty Problemowe Postępów Nauk Rolniczych 296: 1-40. (in Polish) 
SZELEGIEWICZ H. 1978b. Aphids - Aphidodea. Introduction and Lachnidae. Klucze do oznaczania owadów Polski 17(5a): 1-107. (in Polish)

SzELEGIEWICZ H. 1981. The aphids (Homoptera, Aphidoidea) of the Hortobagy. [in:] S. MAHUNKA (ed.). The fauna of the Hortobagy National Park. Volume I. Akadémiai Kiadó, Budapest, 77-87.

SZULCZEWSKI J. 1950. Zoocecidia of the Wielkopolski National Park. Prace Monograficzne nad Przyrodą Wielkopolskiego Parku Narodowego 2: 141-178. (in Polish)

VON DOHLEN C.D., RowE C.A. HEIE O.E. 2006. A test of morphological hypotheses for tribal and subtribal relationships of Aphidinae (Insecta: Hemiptera: Aphididae) using DNA sequences. Molecular Phylogenetic and Evolution 38(2): 316-329.

WATSON G.W. 1982. A biometric, electrophoretic and caryotypic analysis of British species of Macrosiphum (Homoptera; Aphididae). PhD Thesis, University of London, London.

WegIEREK P., WoJCIECHOWSKI W. 2004. Sternorrhyncha. [in:] W. BogDANOWICZ, E. ChudzickA, I. PILIPIUK, E. SkiBińskA (eds.). Fauna Polski - Charakterystyka i wykaz gatunków. T. I. Muzeum i Instytut Zoologii PAN, Warszawa, 234-271.

WIECZOREK K. 2011. Aphid species alien to Poland. Polish Journal of Entomology 80(2): 203-224.

Received: 15 August 2015

Accepted: 29 October 2015 